\title{
Active and Passive Flow Control around Simplified Ground Vehicles
}

\author{
C. H. Bruneau ${ }^{1 \dagger}$, E. Creusé2 ${ }^{2}$ D. Depeyras ${ }^{1}$, P. Gilliéron ${ }^{3}$, I. Mortazavi ${ }^{1}$ \\ ${ }^{1}$ Université Bordeaux, IMB, UMR 5251, F-33400 Talence, France. \\ CNRS, IMB, UMR 5251. INRIA, Team MC2. \\ ${ }^{2}$ LPP (UMR CNRS 8524) Université Lille 1 and Team SIMPAF INRIA Lille Nord Europe, France. \\ ${ }^{3}$ Direction de la recherche RENAULT, France \\ $\dagger$ †orresponding Author Email: bruneau@math.u-bordeaux1.fr or Charles-Henri.Bruneau@math.u-bordeaux1.fr
}

(Received July 16, 2010; accepted March 13, 2011)

\begin{abstract}
The aim of this work is to control the flow around ground vehicles by active or/and passive strategies. The active control is achieved by steady, pulsed or closed-loop jets located at the backof the simplified car model. The passive control is performed using porous layers between the solid body and the fluid in order to modify the shear forces. The two previous control methods can be coupled to improve the drag reduction.
\end{abstract}

Keywords: Numerical simulation, Drag control, Ahmed body.

\section{NOMENCLATURE}

$\begin{array}{llll}C_{d} & \text { Drag coefficient } & \Gamma_{0} & \text { Road } \\ C_{p} & \text { Static pressure coefficient } & \Gamma_{\mathrm{D}} & \text { Entrance section of the domain } \\ C_{p i} & \text { Total pressure coefficient } & \Gamma_{\mathrm{N}} & \text { Artificial frontiers } \\ F_{d} & \text { Drag force } & \Omega & \text { Computational domain } \\ K & \text { Permeability coefficient of the medium } & \partial \Omega & \text { Boundary } \\ p & \text { Pressure } & \rho & \text { Density } \\ U=(u, v, w) & \text { Velocity } & \sigma & \text { Stress tensor }\end{array}$

\section{INTRODUCTION}

With the evolution of human being activities, the saving of energy becomes the main challenge of the next decades. Due to the huge increase of the number of cars and trucks in some countries, the consumption of oil by ground vehicles becomes disturbing and there is a real need to decrease it. When three-dimensional bluff bodies are moving in the vicinity of the ground, they generate a turbulent flow as several separations occur along the body from the front to the back and in the wake. The resulting recirculation zones contribute to a significant part of the drag coefficient, in particular at the back. A way to reduce the drag coefficient of such vehicles is to control the flow around them.

Various active or passive control procedures have been used for decades to control the flow around blunt bodies. Here, two means are studied: a porous layer is added between the fluid and the solid body in order to change the shear forces or actuator jets localized at the right place are used to change the vortex dynamics. The numerical simulations concern the flow around the Ahmed body with or without a rear window on top of a road that models a car or a truck. We first consider the two-dimensional flow around a square back Ahmed body as in this case the flow is mainly two-dimensional. Then, the flow around the Ahmed body with a twentyfive degrees rear window is controlled in threedimensions.

\section{OUTLINE OF THE METHOD}

As it is not easy to consider several media, a global simulation is proposed using the penalization method (Angot et al. (1999), Bruneau and Mortazavi (2008)) which consists to add a term $\mathrm{U} / \mathrm{K}$ in the momentum equation of Navier-Stokes system on the velocity $U$ with $\mathrm{K}$ a non dimensional permeability coefficient. Then it is possible to represent the flow around an obstacle and inside a porous medium as well as in the fluid by changing the value of $\mathrm{K}$. Solving these equations is equivalent to solve the Navier-Stokes equations in the fluid with a Fourier-like boundary condition instead of the no-slip boundary condition. 
Let $H$ be the height of Ahmed body and $\Omega=(0,15 H) \times(0,5 H)$ or $\Omega=(0,12 H) \times(0,6 H) \times(0,4 H)$ be the computational domain, we set $\Omega_{T}=\Omega \times(0, T)$ and $\partial \Omega=\Gamma_{\mathrm{D}} * \Gamma_{0} * \Gamma_{\mathrm{N}}$ where $\Gamma_{\mathrm{D}}$ is the entrance section, $\Gamma_{0}$ is the road and $\Gamma_{\mathrm{N}}$ corresponds to the artificial frontiers (see Fig. 1). The whole problem reads:

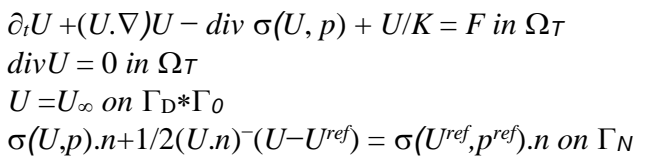

where $\sigma(U, p)=1 / 2 \operatorname{Re}\left(\nabla U+\nabla U^{t}\right)-p I$ stands for the stress tensor, $R e$ is the non dimensional Reynolds number based on $H$ and $\left(U^{r e f}, p^{r e f}\right)$ the reference flow which is taken equal to the flow on the previous cell.

These equations are solved in the whole domain including the solid body which is considered as a porous medium of zero permeability while the fluid is considered as a porous medium of infinite permeability. Numerically, the values are respectively $K=10^{-8}$ and $K=10^{16}$. Furthermore, the porous medium is defined by an intermediate value which has been set to $K=10^{-1}$ to represent an efficient porous medium (Bruneau and Mortazavi (2004)).

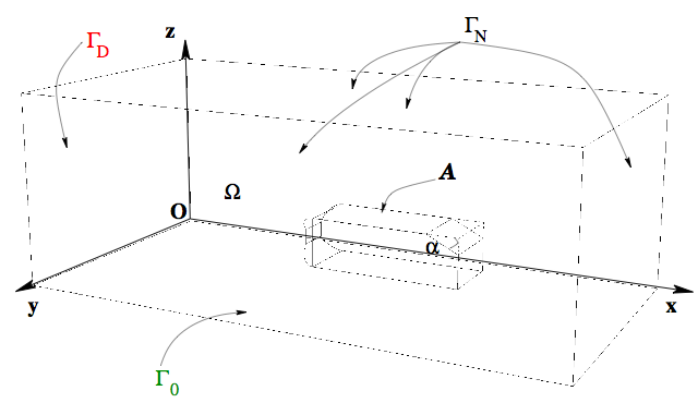

Fig. 1. 3D computational domain around Ahmed body.

The discretization is achieved by a second order Gear scheme in time with explicit treatment of the convection term. All the other terms are considered implicitly. These linear terms are discretized by centered second-order finite differences whereas an upwind third-order Murman-like scheme is used for the convection terms (Bruneau and Saad (2006)). To get a high performance, a multigrid method is applied with a sequence of grids from a coarse $30 \times 10$ cells grid to a fine 1920x640 cells grid in two-dimensions and from a coarse $24 \times 12 \times 8$ cells grid to a fine $768 \times 384 \times 256$ cells grid in three-dimensions. These grids correspond to put respectively 128 and 64 cells on the body height $H$.

This whole method is efficient, accurate and stable enough to make 2D or 3D direct numerical simulations of complex flows. To quantify the control of the flow, some physical quantities are computed. Namely the static pressure coefficient $C_{p}$ and the drag coefficient $C_{d}$. As the flow is computed inside the solid body, we can compute the drag and lift forces by integrating the penalization term on the volume of the body neglecting the time term and the convection term, for instance

$F_{d}=-$ hody $_{\text {biv }} \sigma(U, p) d \Omega \approx$ hody $_{\text {bod }} U / K d \Omega$ where the body includes eventually the porous layers. Then the drag coefficient $C_{d}$ is given by $C_{d}=2 F_{d} / H$ and the static pressure coefficient $C_{p}$ is computed as $C_{p}$ $=2\left(p-p_{0}\right) /\left(\rho|U|^{2}\right)$ where $p_{0}$ is the inlet static pressure that is set to zero at the downstream section of the computational domain. The total pressure coefficient $C_{p i}$ is computed the same way.

\section{CONTROL OF THE SQUARE BACK AHMED BODY FLOW}

For the square back body, the flow separates at the back and is mainly a two-dimensional base flow, almost dominated by the two counter rotating spanwise structures (Gilliéron and Chometon (1999)). So the study on this body is performed only in 2D. For the active control, we insert a blowing jet at the back of the body at $H / 2$. The thickness of the jet corresponds to the height of three cells that is equivalent to $h j=0.024$ and the amplitude of the constant horizontal jet is $u j=$ $0.6 u_{\infty}$. Three different blowing jets with the same amplitude are studied in this work: a steady jet, a periodic pulsed jet (with frequency 0.5 corresponding to the full shedding frequency) and a closed-loop jet. To take into account the shedding on both sides of the back we use for the closed-loop jet, two sensors at H/10 $\left(p_{\text {down }}\right)$ and $9 \mathrm{H} / 10\left(p_{u p}\right)$ and an actuator which velocity is given by $u j=0.6 u\left(1-1.5\left(p-p_{\text {mean }}\right)\right)$ where $p=$ $\min \left(p_{\text {up }}, p_{\text {down }}\right)$ and $p_{\text {mean }}=0.5\left(p_{\text {up }}+p_{\text {down }}\right)$. The three cases have about the same efficiency when the pulsed jet is synchronized to the shedding. The drag coefficient reduction is given on Table 1 .

Table 1 Comparison of the mean drag coefficients for active control with a constant jet, a pulsed jet or a closed-loop jet.

\begin{tabular}{|l|l|l|l|l|l|l|}
\hline Control & $C_{d \text { front }}$ & Var. & $C_{d \text { back }}$ & Var. & $C_{d}$ & Var. \\
\hline No & 0.043 & & 0.084 & & 0.130 & \\
\hline Const. & 0.036 & $-16 \%$ & 0.065 & $-23 \%$ & 0.103 & $-21 \%$ \\
\hline Pulsed & 0.035 & $-19 \%$ & 0.067 & $-20 \%$ & 0.105 & $-19 \%$ \\
\hline Loop & 0.036 & $-16 \%$ & 0.066 & $-21 \%$ & 0.104 & $-20 \%$ \\
\hline
\end{tabular}

The $C_{P}$ profiles on the back wall (Fig. 2) show that the pressure gradient is strongly decreased in the middle and the values are globally much bigger because of the control processes.

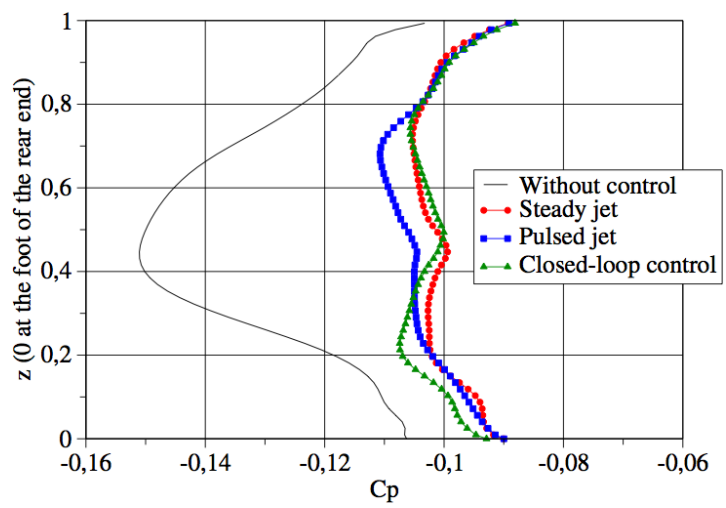

Fig. 2. $C p$ profiles on the back wall without control and with the three control processes. 
In addition to active control a passive control is tested by adding a porous layer in the roof of the body (Fig. 3), modifying the shear forces on the roof and inducing a low speed jet at the upper back expelled into the wake as the flow inside the porous layer is a very slow steady flow. Each phenomenon contributes partially to the modification of the body forces at the back and the down drag is strongly decreased, inducing a significant decrease of the total drag $(-22 \%)$. Also, the $C p$ isolines, as shown on the figure 4 , are less concentrated behind the back wall, meaning that the pressure loss is significantly reduced.

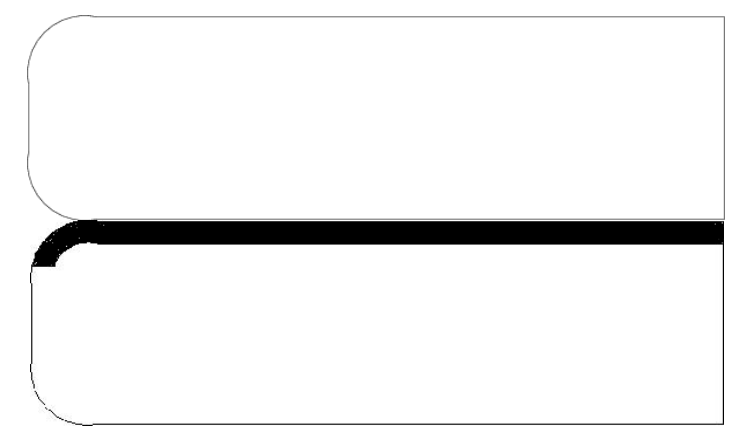

Fig. 3. Genuine square back Ahmed body (top) and body with a porous layer in the roof (bottom).

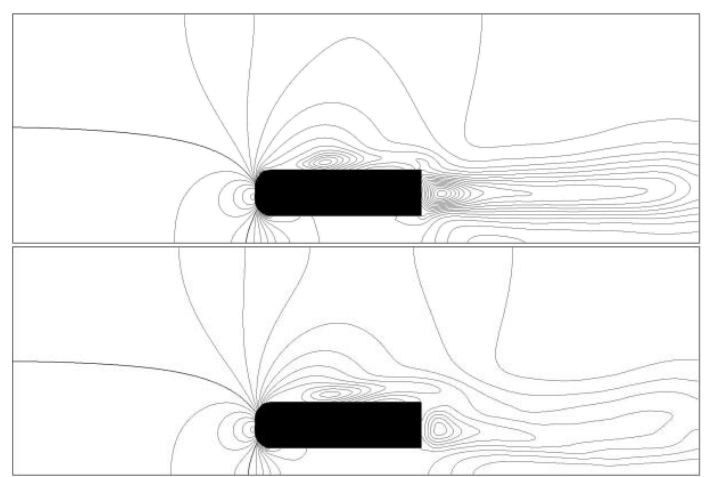

Fig. 4. Comparison of the mean $C_{P}$ isolines for the uncontrolled (top) and the passive controlled (bottom) cases.

The slow Darcy flow in the porous layer induces Kelvin-Helmoltz instabilities that change drastically the size of the vortices on top of the body. This effect is very beneficial to the drag coefficient and so the idea is to couple this effect to an active closed-loop jet at $\mathrm{H} / 3$. The results are summarized in Table 2 . A gain of $31 \%$ is achieved for the coupling that is more than the sum of the two control processes separately.

Table 2 Comparison of the mean drag coefficients for active control with a closed-loop jet at $\mathrm{H} / 3$, a passive device or a coupling of both processes.

\begin{tabular}{|l|l|l|l|l|l|l|}
\hline Control & $C_{d \text { front }}$ & Var. & $C_{d \text { back }}$ & Var. & $C_{d}$ & Var. \\
\hline No & 0.043 & & 0.084 & & 0.130 & \\
\hline Loop & 0.041 & $-5 \%$ & 0.081 & $-4 \%$ & 0.124 & $-5 \%$ \\
\hline Passi. & 0.046 & $+7 \%$ & 0.053 & $-37 \%$ & 0.101 & $-22 \%$ \\
\hline Coupl. & 0.042 & $-2 \%$ & 0.045 & $-46 \%$ & 0.090 & $-31 \%$ \\
\hline
\end{tabular}

It is possible to understand what is exactly going on when coupling the two processes by analysing the $C_{P}$ profiles at the back. With the porous layer there is a strong reduction of the $C_{P}$ in the upper part while the active process there is a significant reduction in the lower part. By coupling the two techniques the $C_{P}$ profile is almost constant all along the back and bigger everywhere than the previous values (Fig. 5).

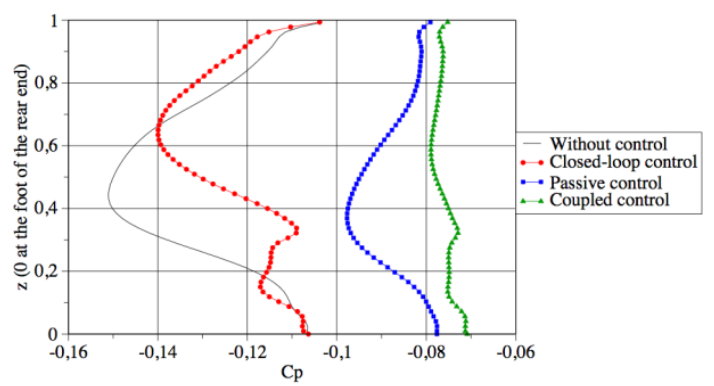

Fig. 5. Comparison of the mean $C p$ profiles on the back wall for the uncontrolled case, the closed-loop active at

$\mathrm{H} / 3$, the passive and the coupled active-passive controlled cases.

\section{CONTROL OF THE 25 DEGREES REAR WINDOW AHMED BODY FLOW}

The second part of this work deals with the 25 degrees rear window Ahmed body. In this case the flow is a genuine three-dimensional flow with longitudinal vortices on both sides of the rear window (Fig. 6). Consequently the results presented are performed in three dimensions.

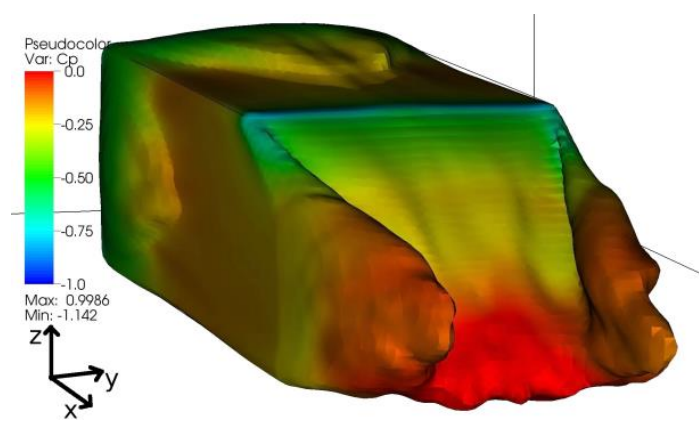

Fig. 6. The mean $C_{P i}$ isosurface colored by $C_{P}$ values.

The main difference with the previous geometry is that it is not possible to put control devices on the rear window except on its boundary. In particular it is not possible to add a porous layer! So, only active controls are studied as a porous layer in the bottom does not decrease significantly the drag coefficient.

The first attempt is to reattach the recirculation zone on the rear window and to push away the structures at the back as in the previous section. Therefore a sucking jet line is set on the top of the rear window and a blowing jet line is set on the middle of the back as shown on Fig. 7. For the sake of simplicity, only constant jets with amplitude 0.6 are studied. The results are encouraging as a significant change in the flow is observed (Fig. 8) according to the goal of this control and there is a drag reduction as shown on Table 3 . 


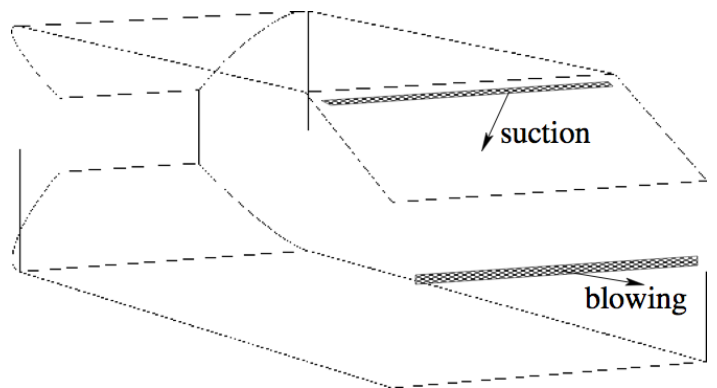

Fig. 7. Transversal control jet lines.

The main effect is obtained on the rear window as the flow is reattached as shown on Fig. 9. The effect of the blowing at the back is less clear. The upper mean vortex is splitted into three different vortices which centers are even closer to the body (Fig. 9). Consequently the drag force is not reduced as much as expected on the back wall.

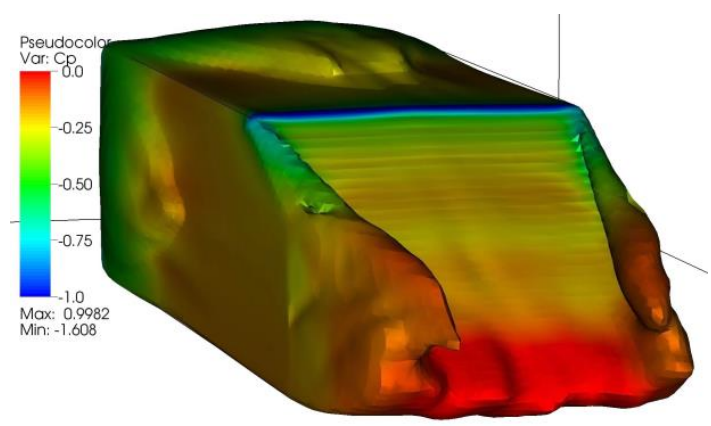

Fig. 8. The mean $C_{P i}$ isosurface colored by $C_{P}$ values for the transversal active control.

Table 3 Comparison of the mean drag coefficients for the transverse and longitudinal active controls and their coupling.

\begin{tabular}{|l|l|l|l|l|}
\hline Control & $C_{d \text { front }}$ & Var. & $C_{d \text { back }}$ & Var. \\
\hline No & 0.177 & & 0.130 & \\
\hline Transverse & 0.176 & $-1 \%$ & 0.125 & $-4 \%$ \\
\hline Longitudinal & 0.164 & $-7 \%$ & 0.130 & \\
\hline Coupling & 0.176 & $-1 \%$ & 0.128 & $-2 \%$ \\
\hline & & & & \\
\hline Control & $C_{d \text { window }}$ & Var. & $C_{d}$ & Var. \\
\hline No & 0.149 & & 0.371 & \\
\hline Transverse & 0.132 & $-11 \%$ & 0.345 & $-7 \%$ \\
\hline Longitudinal & 0.123 & $-17 \%$ & 0.332 & $-11 \%$ \\
\hline Coupling & 0.118 & $-21 \%$ & 0.323 & $-13 \%$ \\
\hline
\end{tabular}

The second attempt is to reduce the intensity of the longitudinal vortices adding a longitudinal jet line on both sides of the rear window (Fig. 10). Here again the effect of the control is close to our attempts as there is a strong decrease of the drag coefficient on the rear window and even in front of the body (Table 3). The size of the longitudinal vortices is increased by the blowing but their strength and their effect on the body are decreased significantly (Fig. 11). The effect is very clear on the $C p$ profiles presented in Fig. 12, the $C p$ values are much higher on the top of the rear window ( 0 corresponds to the bottom and 1 to the top). Indeed the mean value in the upper half on the edge goes from 0.55 to -0.34 . Consequently the gain of drag coefficient reaches $11 \%$ (Table 3 ).
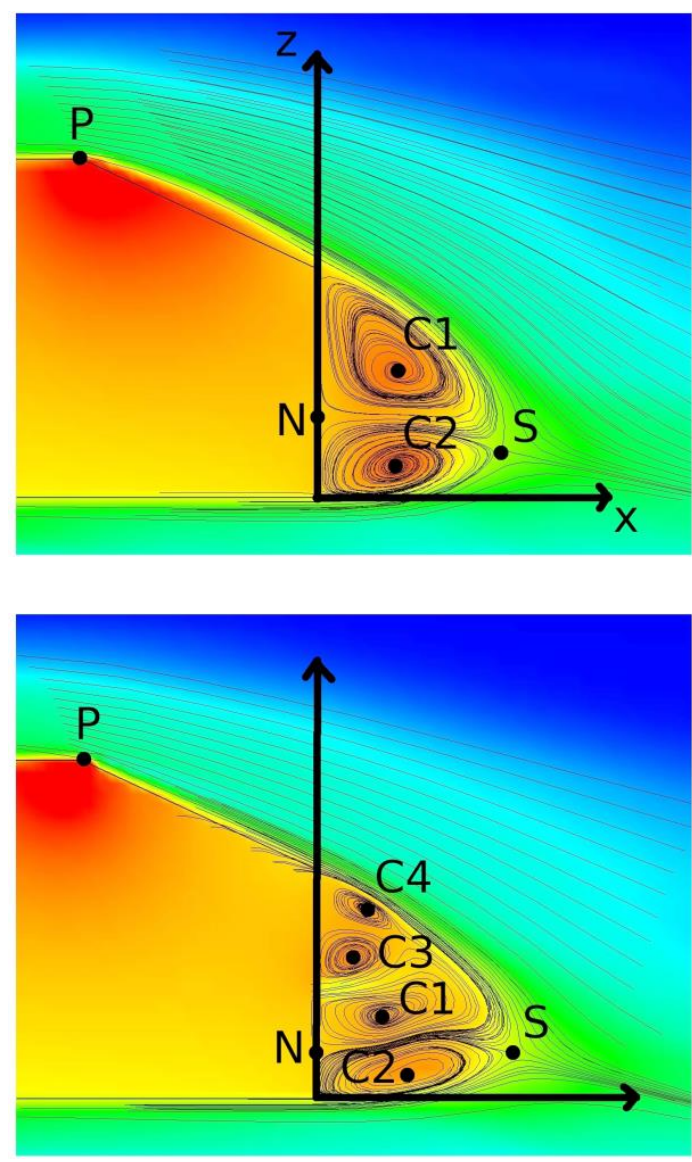

Fig. 9. Mean streamlines in the median y plane colored by the mean $C_{p i}$ values for the uncontrolled (top) and the transversal active controlled (bottom) cases.

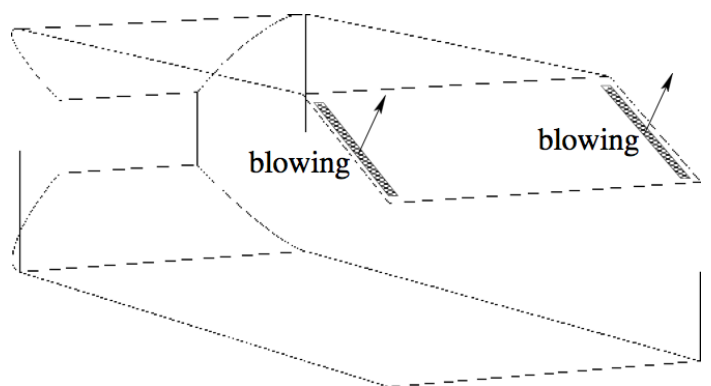

Fig. 10. Longitudinal control jet lines.

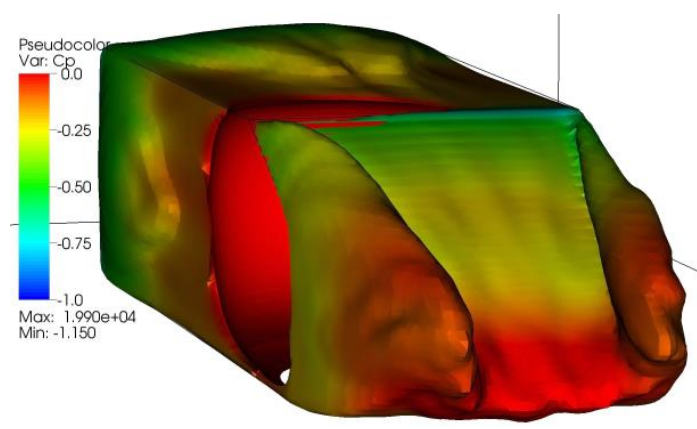

Fig. 11. The mean $C_{P i}$ isosurface colored by $C_{P}$ values for the longitudinal active control. 


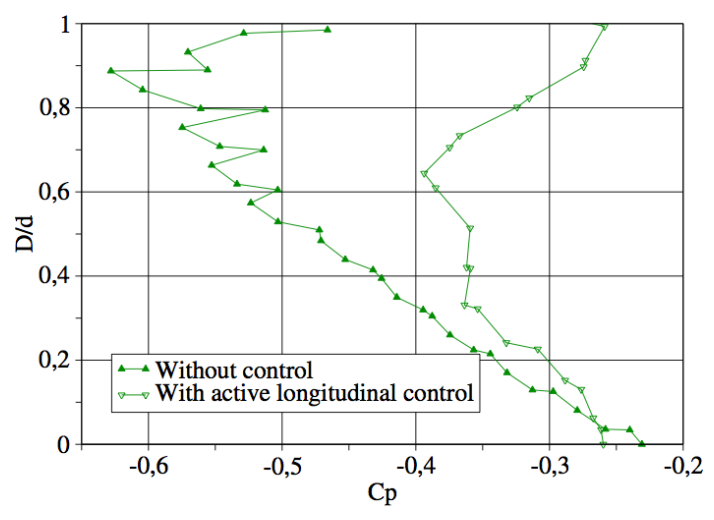

Fig. 12. Mean $C_{P}$ profile on the edge of the rear window for the uncontrolled and the longitudinal active controlled cases.

Finally a coupling of the two active control processes is proposed. The goal is to reattach the flow in the middle of the rear window and to decrease the influence of the longitudinal vortices to the body. In terms of drag coefficient the results are given in Table 3 . They show that both control processes are very efficient on the rear window as the decrease is higher than $20 \%$ on this part. Unfortunately, as said above the results on the back are not so good and consequently the total drag reduction is $13 \%$. The Fig. 13 shows that the $C_{P}$ profile on the back is not much improved except at the bottom as the vortex is reduced and its centre $\mathrm{C} 2$ is slightly pushed away (Fig. 9). The other centres are closer to the wall and even if there are smaller than the initial vortex, they induce an equivalent drag force on the wall. To improve this result we need to understand precisely the vortex dynamics at the back and how it can be positively modified by the control.

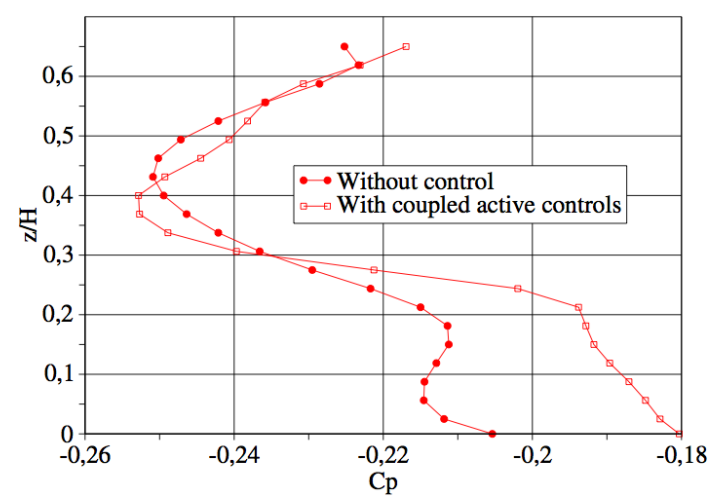

Fig. 13. Mean $C_{P}$ profile on the middle of the back for the uncontrolled and the coupled active control cases.

\section{CONClusions}

Various passive and active control processes are proposed to reduce the drag coefficient of Ahmed body. For the square back Ahmed body it is possible to couple the two kinds of control to reach a very good drag reduction adding the beneficial effects of both processes. For the 25 degrees rear window body, a very good reduction of the drag forces is achieved on the window with active control using succing and blowing jet lines.

\section{ACKNOWLEDGEMENTS}

This research was partly supported by Renault car industry.

\section{REFERENCES}

Angot, Ph., Ch.-H. Bruneau, and P. Fabrie (1999). A penalization method to take into account obstacles in incompressible viscous flows. Numer. Math. 81.

Bruneau, Ch.-H., and I. Mortazavi (2004). Passive control of bluff body flows using porous media. Int. J. for Num. Meth. in Fluids 46.

Bruneau, Ch.-H., and I. Mortazavi (2008). Numerical modelling and passive flow control using porous media. Computers \& Fluids 37 (5).

Bruneau, Ch.-H., and M. Saad (2006). The 2D liddriven cavity problem revisited. Computers \& Fluids 35 (3).

Gilliéron, P., F. Chometon (1999). Modelling of Stationary Three-Dimensional Separated Air Flows around an Ahmed Reference Model. ESAIM 7. 